\title{
Sodium Iodate Selectively Injuries the Posterior Pole of the Retina in a Dose-Dependent Manner: Morphological and Electrophysiological Study
}

\author{
Anna Machalińska • Wojciech Lubiński • Patrycja Kłos • Miłosz Kawa • \\ Bartłomiej Baumert · Krzysztof Penkala • Ryszard Grzegrzółka • \\ Danuta Karczewicz • Barbara Wiszniewska - Bogusław Machaliński
}

Accepted: 10 August 2010/Published online: 20 August 2010

(C) The Author(s) 2010. This article is published with open access at Springerlink.com

\begin{abstract}
Sequential morphological and functional features of retinal damage in mice exposed to different doses $(40$ vs. $20 \mathrm{mg} / \mathrm{kg})$ of sodium iodate $\left(\mathrm{NaIO}_{3}\right)$ were analyzed. Retinal morphology, apoptosis (TUNEL assay), and function (electroretinography; ERG) were examined at several time points after $\mathrm{NaIO}_{3}$ administration. The higher dose of $\mathrm{NaIO}_{3}$ caused progressive degeneration of the whole retinal area and total suppression of scotopic and photopic ERG. In contrast, the lower dose induced much less severe degeneration in peripheral part of retina along with a moderate decline of $b$ - and a-wave amplitudes in ERG, corroborating the presence of regions within retina that retain their function. The peak of photoreceptor apoptosis was found on the 3 rd day, but the lower dose induced more intense reaction within the central retina than in its peripheral region. In conclusion, these results indicate that peripheral area of the retina reveals better resistance to $\mathrm{NaIO}_{3}$ injury than its central part.
\end{abstract}

\footnotetext{
A. Machalińska · B. Wiszniewska

Department of Histology and Embryology, Pomeranian Medical University, Al. Powstancow Wlkp. 72, 70-111 Szczecin, Poland e-mail: annam@sci.pam.szczecin.pl
}

A. Machalińska · W. Lubiński · K. Penkala · D. Karczewicz Department of Ophthalmology, Pomeranian Medical University, Szczecin, Poland

\author{
A. Machalińska · P. Kłos · B. Baumert · R. Grzegrzółka · \\ B. Machaliński ( $\square)$ \\ Department of General Pathology, Pomeranian Medical \\ University, Szczecin, Poland \\ e-mail: machalin@sci.pam.szczecin.pl
}

\section{Kawa}

Department of Physiology, Pomeranian Medical University, Al. Powstancow Wlkp. 72, 70-111 Szczecin, Poland
Keywords Apoptosis - Electroretinogram $\cdot$ Retinal degeneration $\cdot$ Sodium iodate

\section{Introduction}

The retinal pigment epithelium (RPE) is a monolayer of pigmented cuboidal cells that perform highly specialized and unique functions essential for homeostasis of the neural retina. Located between vessels of the choriocapillaris and light-sensitive outer segments of the photoreceptors, the RPE closely interacts with photoreceptors in maintaining the visual process. The RPE provides multiple functions that support normal photoreceptor activity. These include the phagocytosis of outer segments of photoreceptors, the directional transport of nutrients, removal of waste products from photoreceptor cells, as well as visual pigment transport and regeneration. In addition, the RPE is able to secrete a variety of growth factors that are essential for maintaining the structural integrity of the retina [1]. Thus RPE damage caused by environmental factors and/or genetic mutations leading to a failure of any of its functions can contribute to degeneration of the retina, visual impairment, and blindness.

There is a wide spectrum of retinal disorders in which the primary or predominant site of dysfunction is localized in the retinal pigment epithelium or adjacent photoreceptors. Retinitis pigmentosa (RP) comprises a group of eye disorders with a complex molecular etiology affecting both the RPE and photoreceptors. Despite genetic heterogeneity, patients with RP tend to have a common clinical pattern of diffuse or patchy areas of retinal atrophy with vascular attenuation and pigmentary clumping [2]. There is a great number of animal models available for studying these diseases. Systemic administration of sodium iodate $\left(\mathrm{NaIO}_{3}\right)$ is known to selectively impair the RPE, resulting in patchy loss of RPE 
and subsequent degeneration of photoreceptors. $\mathrm{NaIO}_{3}-$ induced retinal toxicity has been reported in a variety of animal species, including rabbits, sheep, rats, and mice [3-6].

Although $\mathrm{NaIO}_{3}$ has been used extensively as a retinotoxin, there are only single reports concerning a precise sequential morphological time-dependent observation of changes in the retinal pigment epithelium in response to different doses of $\mathrm{NaIO}_{3}$. However, the pathogenesis of RPE cell destruction induced by sodium iodate still requires clarification, particularly in the field of kinetics of morphologic changes and programmed cell death within different areas of the retina as well as their strict correlation with retinal functional response. Elucidation of these aspects would provide better understanding of the chemical model of RPE damage and consequently lead to its widespread use in the preclinical trials aimed at introducing more effective therapies for various ocular degenerative disorders in humans. Thus in the present study we examined the morphologic and functional characteristics of $\mathrm{NaIO}_{3}$-induced retinal damage in mice with particular interest in the progression of RPE destruction in the central and peripheral parts of an eyeball. We also focused on the subsequent degeneration of the outer layers of the neurosensory retina. To address this issue, we investigated the effects of $\mathrm{NaIO}_{3}$ administrated in two different concentrations, i.e. 40 and $20 \mathrm{mg}$ per $\mathrm{kg}$ of the body mass. Morphological changes, apoptosis induction, and electrophysiological function of the retina were determined at several time points after each dose administration.

\section{Materials and Methods}

Mice

Pathogen-free 8- to 12-month-old mature male C57BL mice (Polish Academy of Sciences, Wroclaw, Poland) weighing 27-29 g were used in the experiment. The mice were housed in a standard laboratory environment with a 12-h/12-h light-dark cycle at $21^{\circ} \mathrm{C}$. All experimental procedures involving animals were performed according to the regulations in the ARVO Statement for the Use of Animals in Ophthalmic and Vision Research and were approved by the local ethics committee.

$\mathrm{NaIO}_{3}$ Injection

Sodium iodate (Sigma, USA) was diluted in sterile phosphate-buffered saline (PBS) to concentrations of 5 and $2.5 \mathrm{mg} / \mathrm{mL}$ and stored at $4^{\circ} \mathrm{C}$. Mice from each experimental group were injected via the orbital venous plexus with two different $\mathrm{NaIO}_{3}$ doses: a higher $(40 \mathrm{mg} / \mathrm{kg})$ and a lower $(20 \mathrm{mg} / \mathrm{kg})$. Control mice were injected with physiological saline instead of $\mathrm{NaIO}_{3}$.
Histology

Mice (5 animals at each time point and dose) were euthanatized $1,3,7,18$, or 28 days after $\mathrm{NaIO}_{3}$ administration, by cervical dislocation. The eyes were then enucleated and fixed in $4 \% \mathrm{PFA}$ at $4^{\circ} \mathrm{C}$ overnight. For crosssections, the eyes were embedded in paraffin, cut into 5-micrometer-thick sections, and stained with hematoxylin and eosin (Sigma). Morphological analyses including measurements of the outer nuclear layer as well as IHC studies were performed at two different locations in each eye: approx. $300 \mu \mathrm{m}$ from the optic nerve head for the central retina (posterior pole) and approx. $300 \mu \mathrm{m}$ from the ora serrata for the peripheral retina. Whole eye flat mounts (FMs) were prepared by removing the anterior segment and the neurosensory retina from the eyeball followed by making four radial relaxing incisions in the remaining sclera-choroid-RPE complex. The specimens (FMs) were mounted with DAPI mounting medium (Vectashield ${ }^{\mathrm{TM}}$ Hard-Set with DAPI, Vector Laboratories, Burlingame, CA, USA), coverslipped, and visualized with the use of a BD Pathway $^{\text {TM }} 855$ (BD Biosciences, Rockville, MD, USA). The fluorescence emission was collected in the green channel $(515 \mathrm{~nm})$ for RPE autofluorescence and in the blue channel $(435 \mathrm{~nm})$ for DAPI. During the processing of the images, the autofluorescence channel was pseudocolored green, whereas the nuclei were pseudocolored blue.

\section{Detection of Apoptosis}

The eye sections were deparaffinized in xylene $(2 \times 15 \mathrm{~min})$ followed by hydration in decreasing ethanol solutions $(100,95,85,70$, and 50\%). They were subsequently rinsed in distilled water (for $2 \mathrm{~min}$ ) and subjected to apoptosis detection procedures. The TUNEL assay was performed by means of a TdT In Situ-DAB in situ Apoptosis Detection Kit (R\&D Systems, McKinley Place, MN, USA) according to the manufacturer's instructions. The specimens were examined under a light microscope (Axioskop, Carl Zeiss, Jena, Germany) with an objective magnification of $40 \times$ and images were captured with a Power Shot A640 digital camera (Canon, Shanghai, China). The apoptotic (TUNEL-positive) nuclei per visual field were counted in at least three visual fields per each central and peripheral part of the retina using AxioVision Rel. 4.8 software (Carl Zeiss, Jena, Germany).

\section{ERG Recordings}

Scotopic and photopic ERGs were recorded 1, 3, 7, 18, and 28 days after the sodium iodate administration. After 4-hlong dark adaptation, mice were anesthetized with an intraperitoneal injection of ketamine $(40 \mathrm{mg} / \mathrm{kg})$ and xylazine 
( $4 \mathrm{mg} / \mathrm{kg}$ ). Then the cornea was anesthetized (Alcaine; Alcon) and the pupils were dilated with $1 \%$ atropine. Body temperature was maintained using a heating pad. Retinal responses were recorded monocularly with the use of a gold ring contact electrode (LKC Technologies, Gaithersburg, USA). Needle electrodes placed under the scalp between the eyes and in the tail served as the reference and ground leads, respectively. ERGs were differentially amplified $(0.05-1,500 \mathrm{~Hz})$, averaged, and stored using an LKC UTAS E-2000 system. ERGs were recorded in response to strobe flash stimuli presented in the LKC Ganzfeld bowl, similar to the equipment used for human testing.

For the assessment of rod photoreceptor function, a strobe white-flash stimulus was presented to the darkadapted dilated eye with a low flash intensity $(24 \mathrm{~dB}$ attenuation) and eight responses recorded in an interval of $8 \mathrm{~s}$ were computer averaged. Mixed rod and cone responses were obtained using stimulation with white flashes of maximum intensity equal to approximately $1.6 \mathrm{~cd} \times \mathrm{s} / \mathrm{m}^{2}$ (Standard Flash, SF, $0 \mathrm{~dB}$ attenuation). The retinal responses were measured twice with a 28 -s interstimulus interval and averaged. To evaluate the function of cone photoreceptors, animals were light-adapted for $10 \mathrm{~min}$ under a white background $\left(32 \mathrm{~cd} / \mathrm{m}^{2}\right)$. After that, a strobe white-flash stimulus was presented to the dilated eye in the Ganzfeld bowl using maximum flash intensity ( $0 \mathrm{~dB}$ attenuation) and responses to 8 flashes with an interval of $1 \mathrm{~s}$ were recorded and averaged. The amplitude of the b-wave was measured from the a-wave trough to the peak of the b-wave or, if not a-wave was present, from the prestimulus baseline to the peak of the b-wave. The amplitude of the a-wave was measured from the prestimulus baseline to the peak of the a-wave.

\section{Data Analysis}

Mean values \pm SD were calculated from the data obtained from 50 experimental mice $(5$ mice at each time point and $\mathrm{NaIO}_{3}$ dose) and 20 control mice using Statistica 5.0 software (Chicago, IL, USA). The results were then analyzed by the Mann-Whitney $U$ test and a p value less than 0.05 was considered statistically significant.

\section{Results}

\section{Retinal Histopathology}

$\mathrm{NaIO}_{3}$ is a well-known retinotoxin that selectively injures the RPE with secondary effects on photoreceptors. In our study we found the $\mathrm{NaIO}_{3}$-induced structural and functional changes to be time and dose dependent. To provide more specific analysis we firstly employed conventional and the most commonly used dose of $\mathrm{NaIO}_{3}$, i.e. $40 \mathrm{mg} / \mathrm{kg}$ and additionally the dose reduced by one-half $(20 \mathrm{mg} / \mathrm{kg})$. The retinas of sodium iodate-treated mice as well as control retinas were examined $1,3,7,18$, and 28 days after $\mathrm{NaIO}_{3}$ administration. Retinal morphology as well as apoptotic cells visualized with TUNEL assay were evaluated by a light microscopy. Histopathological changes within the retinal pigment epithelium were precisely analyzed based on autofluorescence of RPE flat mounts examined by fluorescence microscopy.

When using the higher dose of $\mathrm{NaIO}_{3}(40 \mathrm{mg} / \mathrm{kg})$ we observed progressive degeneration of the neurosensory retina along with widespread loss of RPE cells. These changes spread through the posterior pole and the peripheral region, indicating significant damage within the whole retinal area. On the first day after $\mathrm{NaIO}_{3}$ administration we discerned marked RPE damage with slight changes in the neurosensory retina in the form of some disorganization of the outer and inner segments of the photoreceptors (Fig. 1c). Individual RPE cells were flattened and most were devoid of nuclei, although they maintained their orientation and boundaries. On the third day after $\mathrm{NaIO}_{3}$ injection we detected complete destruction of the RPE, which had been replaced by a thin layer of melanin released along Bruch's membrane (Fig. 2c). At this time point, the retinal pigment epithelium FMs revealed a discontinuous melanin sheet displaying empty spaces with nuclei present, possibly belonging to inflowing cells (Fig. 2d). Discontinuity of the RPE layer, macrophage infiltration, and disrupted structure of the outer and inner photoreceptor segments were observed in the retinal H-E stained sections (Fig. 2c). Moreover, TUNEL staining revealed the presence of massive apoptosis in the outer nuclear layer, which corroborated immense degeneration of the photoreceptors (Fig. 2b). By the 7th day after $\mathrm{NaIO}_{3}$ delivery, the thickness of the outer nuclear layer decreased significantly and the outer and inner photoreceptor segments were markedly shortened (Fig. 1e). The RPE flat mount obtained at this time point revealed an irregular network of melanin remnants spread along Bruch's membrane (Fig. 1d). On the 18th and 28th days after $\mathrm{NaIO}_{3}$ administration we observed bumpy melanin clumping scattered along Bruch's membrane with no apparent RPE cover. There were also features suggesting that glial cells might contribute to the phagocytosis of melanin remnants (Fig. 1f). The retinal thickness was drastically reduced, predominantly due to the progressive photoreceptor loss.

The lower concentration of $\mathrm{NaIO}_{3}(20 \mathrm{mg} / \mathrm{kg})$ showed much less severe damage to the eye tissues. Damage to the RPE and photoreceptor degeneration were most pronounced in the central region of the retina, with relative sparing of the peripheral retina adjacent to the ora serrata (Fig. 2f, g). Additionally, the lower dose of $\mathrm{NaIO}_{3}$ led to a lessening of RPE and retinal degeneration in the peripheral 
Fig. 1 Representative images visualizing histopathological changes within the central part of the retina recorded 1 (c), 7 (d, e), and 28 (f) days after $\mathrm{NaIO}_{3}$ injection in both the higher and lower doses;

(a, b) saline-treated control mice. Retinal morphology was analyzed in the whole eye flat mounts (FMs) with RPE nuclei pseudocolored blue and green indicating RPE

autofluorescence (a and $\mathbf{d}$ ) as well as in $\mathrm{H} / \mathrm{E}$ stained sections (b, c, e, and $\mathbf{f}$ ). The black arrow (f) indicates glial (Müller) cells phagocyting melanine remnants. $G C L$ ganglion cell layer, $I N L$ inner nuclear layer, $O N L$ outer nuclear layer
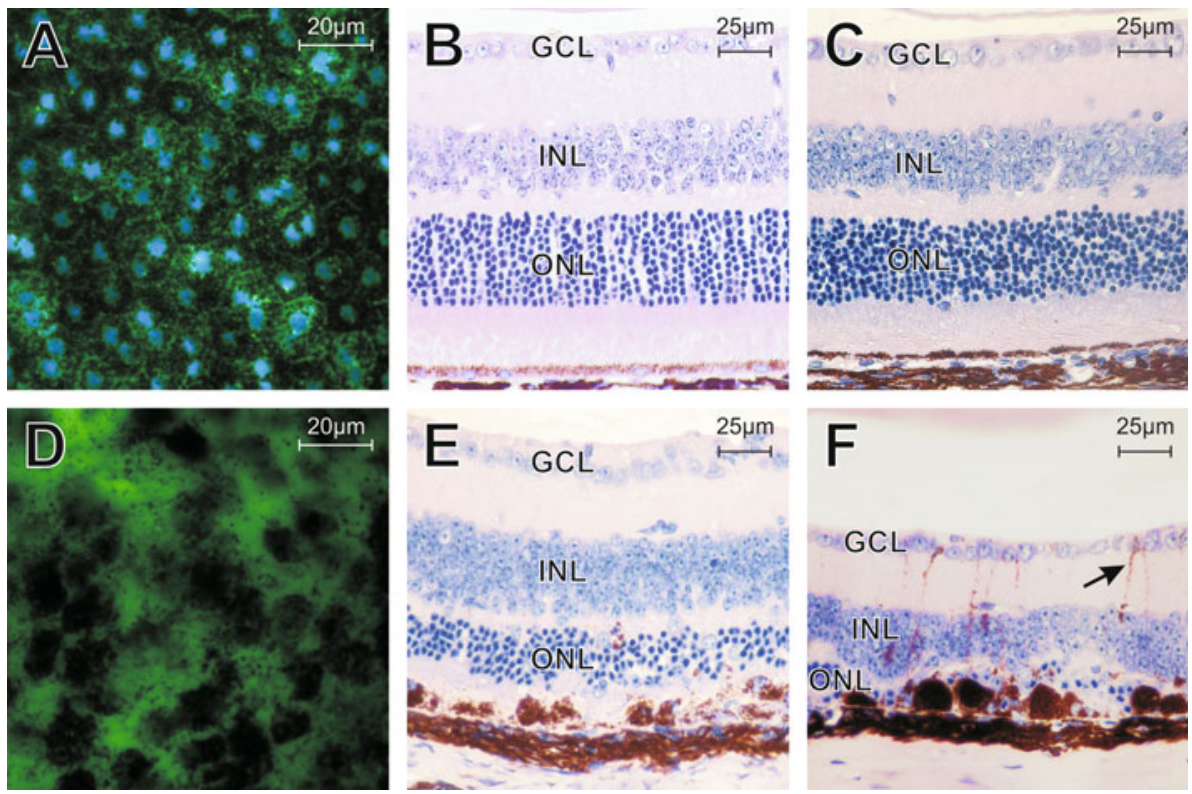

retina, whereas the histopathological changes within its central region were similar to those observed after the injection of the higher dose of $\mathrm{NaIO}_{3}$. The histological analyses, including measurements of outer nuclear layer thickness both in peripheral and central parts of the retina, are summarized in Fig. 3. Of note, a peak of photoreceptor cell death identified by a TUNEL assay was observed on the 3rd day after $\mathrm{NaIO}_{3}$ administration in both the lower and higher dose models, indicating the time point when the most significant damage within the outer nuclear layer occurred (Fig. 2h, i). What is more, based on the results obtained from the TUNEL method, we found a regional pattern in the form of a more intense apoptotic reaction visible within the central part of the retina than in its peripheral region (Fig. 2a). The morphological changes involving those present in the RPE as well as in photoreceptors are summarized in Table 1.

\section{Electrophysiology}

The flash electroretinogram (ERG) is a valuable tool for assessing retinal function and represents the summed activity of retinal cells. In order to perform a more quantitative analysis of both rods and cones response to RPE and neurosensory retina destruction we registered ERG at different time points after $\mathrm{NaIO}_{3}$ administration in the higher and lower doses. Figure 4a illustrates a typical ERG obtained from control (saline-treated) mice. A rod-specific response was elicited by a dim white flash after dark adaptation and is characterized by a prominent positivegoing b-wave arising in the inner nuclear layer. The standard bright white-flash response recorded under scotopic conditions represents a combined rod-cone response. This response contains a negative a-wave generated by photoreceptor hyperpolarization and a positive-going b-wave reflecting post-phototransduction or post-receptoral function. After restoration of photopic conditions (rod-suppressing background illumination), the recorded photopic responses reflect cone system activities.

The changes in the scotopic and photopic ERGs recorded at different time points after $40 \mathrm{mg} / \mathrm{kg} \mathrm{NaIO}$ injection are summarized in Fig. 4. In the rod ERG response performed on the 1st day after $\mathrm{NaIO}_{3}$ administration, the b-wave amplitudes were significantly reduced, indicating severe rod system impairment. In the scotopic bright-flash combined rod-cone response at this time point we observed that the b-wave amplitude decreased more rapidly than that of the $\mathrm{a}$-wave, which resulted in a b/a amplitude ratio $<1.0$. This characteristic pattern of waveform changes can be defined as an electronegative or "negative" ERG. For the cone ERG response performed on the 1 st day after $\mathrm{NaIO}_{3}$ delivery, the b-wave amplitudes were slightly reduced without profound waveform alterations, suggesting a relative high cone system preservation. As early as the 3rd day after $\mathrm{NaIO}_{3}$ injection, both the scotopic and photopic responses were abolished and became undetectable, indicating advanced functional damage to the whole retinal area. Functional recovery of the retina was not achieved on successive days (7th, 18th, and 28th days) following $\mathrm{NaIO}_{3}$ injection.

In contrast to the higher dose, the lower concentration of $\mathrm{NaIO}_{3}$ led to moderate decrease in the b-wave and a-wave amplitudes under both scotopic and photopic conditions, but not to their complete extinction, suggesting the presence of less damaged regions of the retina. The attenuation in the ERG response was stable for 28 days after sodium iodate delivery, as shown on Fig. 5. 

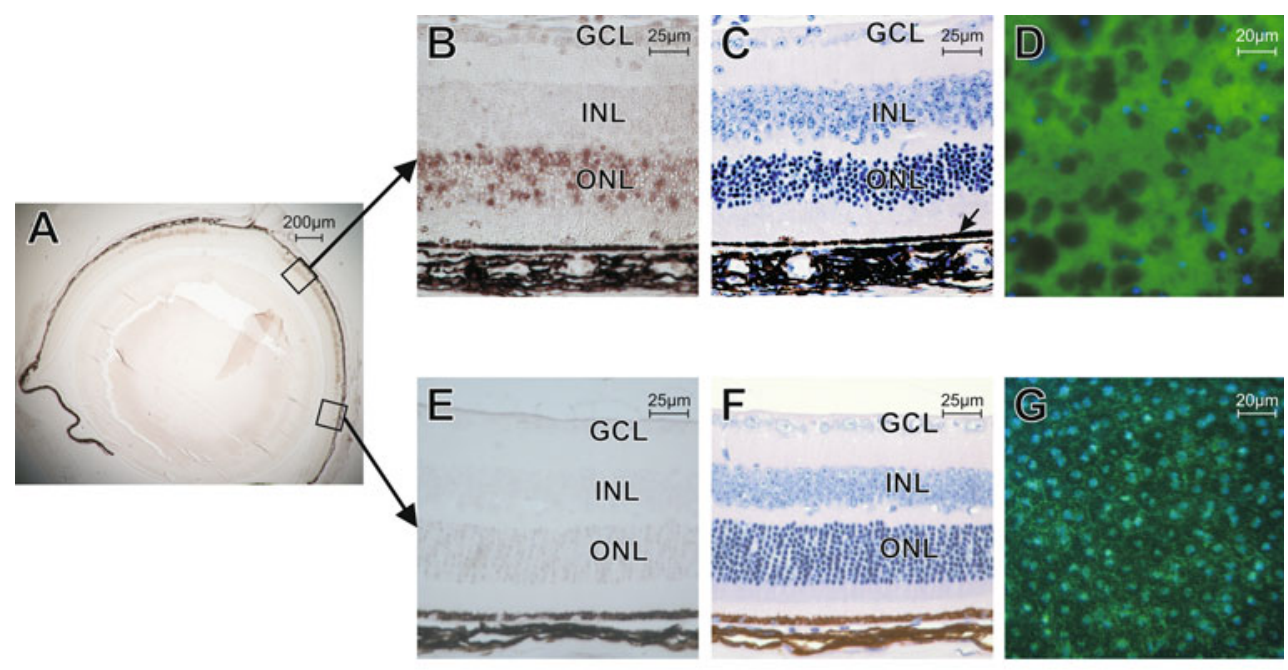

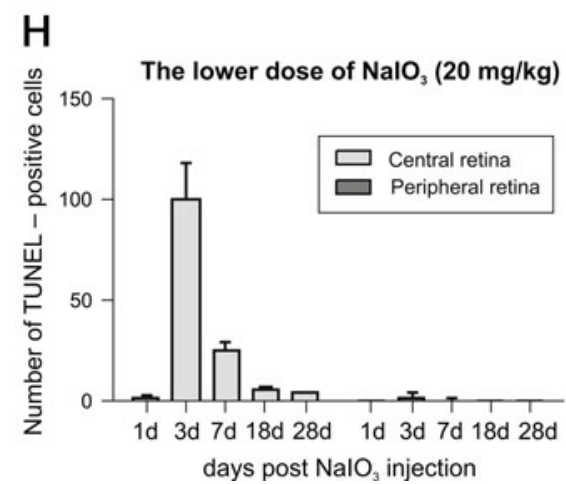

Fig. 2 The regional pattern of retinal damage after the injection of the lower dose $(20 \mathrm{mg} / \mathrm{kg})$ of sodium iodate (a). TUNEL-stained sections visualizing apoptotic photoreceptors located in the central (b) and peripheral (e) parts of the retinal area on the 3rd day post $\mathrm{NaIO}_{3}$ exposure. (c, f) corresponding (central vs. peripheral part) H/E stained sections. (d, g) corresponding (central vs. peripheral part) whole eye flat mounts (FMs) with nuclei pseudocolored blue; green pseudocolor indicates RPE autofluorescence. The black arrow

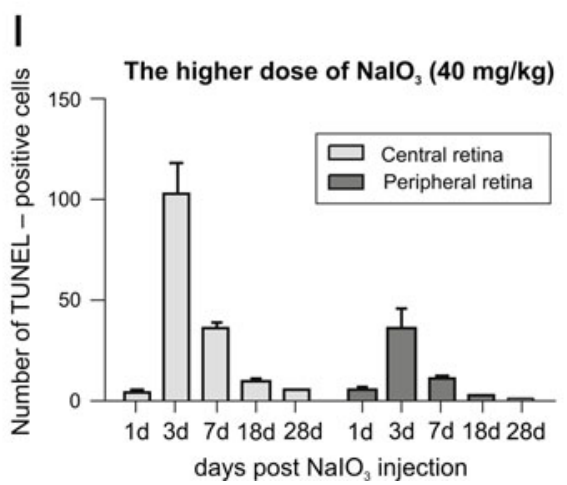

(c) indicates a thin layer of melanin released along Bruch's membrane. The graphs illustrate the difference in the number of apoptotic cells between the central and peripheral parts of the retina exposed to the lower (h) and higher (i) doses of $\mathrm{NaIO}_{3}$. Photoreceptor apoptosis shows the peak intensity on the 3rd day after $\mathrm{NaIO}_{3}$ administration regardless of the dose used. $G C L$ ganglion cell layer, $I N L$ inner nuclear layer, $O N L$ outer nuclear layer
Fig. 3 A comparison of outer nuclear layer thickness $(\mu \mathrm{m}$; mean $\pm \mathrm{SD}$ ) at the different time points after $\mathrm{NaIO}_{3}$ administration at the higher $(40 \mathrm{mg} / \mathrm{kg})$ and lower $(20 \mathrm{mg} / \mathrm{kg})$ dose. a The central part of the retina (posterior pole), approx. $300 \mu \mathrm{m}$ from the optic nerve head. b The peripheral part of the retina, approx. $300 \mu \mathrm{m}$ from the ora serrata. $* P<0.05$ lower versus higher dose

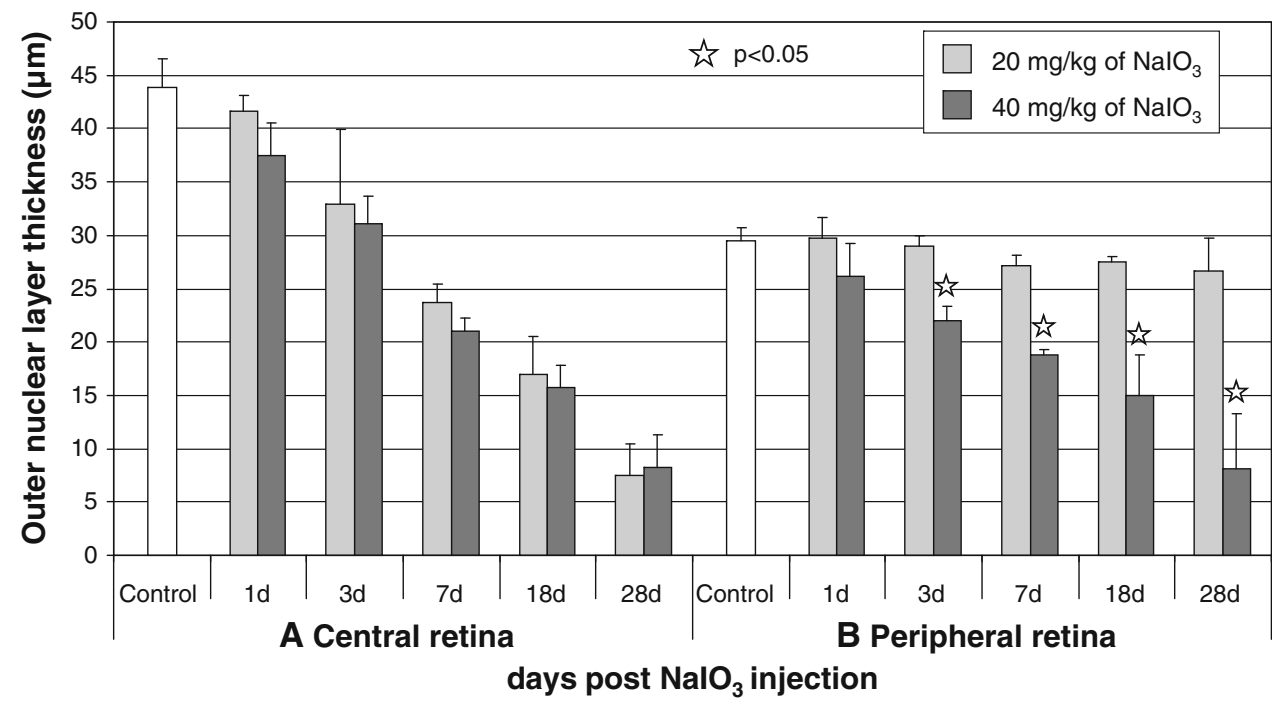


Table 1 The pathological changes within the central and peripheral retina exposed to two different doses of $\mathrm{NaIO}_{3} ; 1,3,7,18,28$ days after $\mathrm{NaIO}_{3}$ administration

\begin{tabular}{|c|c|c|c|c|}
\hline \multirow{2}{*}{$\begin{array}{l}\text { Days post } \\
\text { injection }\end{array}$} & \multicolumn{2}{|l|}{ Central retina } & \multicolumn{2}{|l|}{ Peripheral retina } \\
\hline & $\begin{array}{l}\text { Higher dose of } \mathrm{NaIO}_{3} \\
(40 \mathrm{mg} / \mathrm{kg})\end{array}$ & $\begin{array}{l}\text { Lower dose of } \mathrm{NaIO}_{3} \\
(20 \mathrm{mg} / \mathrm{kg})\end{array}$ & $\begin{array}{l}\text { Higher dose of } \mathrm{NaIO}_{3} \\
(40 \mathrm{mg} / \mathrm{kg})\end{array}$ & $\begin{array}{l}\text { Lower dose of } \mathrm{NaIO}_{3} \\
(20 \mathrm{mg} / \mathrm{kg})\end{array}$ \\
\hline 1 & \multicolumn{3}{|c|}{$\begin{array}{l}\text { Marked RPE damage-kariolysis, disorganization of the outer and inner } \\
\text { photoreceptor segments, no apparent changes in the outer nuclear layer }\end{array}$} & $\begin{array}{l}\text { No significant morphological } \\
\text { changes in the RPE and adjacent } \\
\text { neurosensory retina }\end{array}$ \\
\hline 3 & \multicolumn{3}{|c|}{$\begin{array}{l}\text { Complete destruction of the RPE, disrupted structure of outer and inner } \\
\text { photoreceptor segments, massive apoptosis observed in the outer nuclear layer }\end{array}$} & $\begin{array}{l}\text { No significant morphological } \\
\text { changes in the RPE and adjacent } \\
\text { neurosensory retina }\end{array}$ \\
\hline 7 & \multicolumn{3}{|c|}{$\begin{array}{l}\text { Melanin remnants spread along Bruch's membrane, decreased outer nuclear layer } \\
\text { thickness, markedly shortened outer and inner photoreceptor segments }\end{array}$} & $\begin{array}{l}\text { No significant morphological } \\
\text { changes in the RPE and adjacent } \\
\text { neurosensory retina }\end{array}$ \\
\hline 18 & \multirow{2}{*}{\multicolumn{3}{|c|}{$\begin{array}{l}\text { Bumpy melanin clumping scattered along Bruch's membrane, outer nuclear layer } \\
\text { thickness drastically reduced }\end{array}$}} & No significant morphological \\
\hline 28 & & & & $\begin{array}{l}\text { changes in the RPE and adjacent } \\
\text { neurosensory retina }\end{array}$ \\
\hline
\end{tabular}

Fig. 4 ERG responses recorded at different time points after $\mathrm{NaIO}_{3}$ administration in the higher dose $(40 \mathrm{mg} / \mathrm{kg})$. a saline-treated control mice. b ERG recorded on the 1st day after sodium iodate delivery. c ERG recorded on the 3rd day after sodium iodate injection. d The b-wave amplitude measurements on the $1 \mathrm{st}, 3 \mathrm{rd}$, 7th, 18th, and 28th day after $\mathrm{NaIO}_{3}$ administration $(\mu \mathrm{V}$; mean $\pm \mathrm{SD}$ ). From the 3rd day after $\mathrm{NaIO}_{3}$ injection, both the scotopic and photopic responses were abolished and became undetectable. $* P<0.05$ versus control
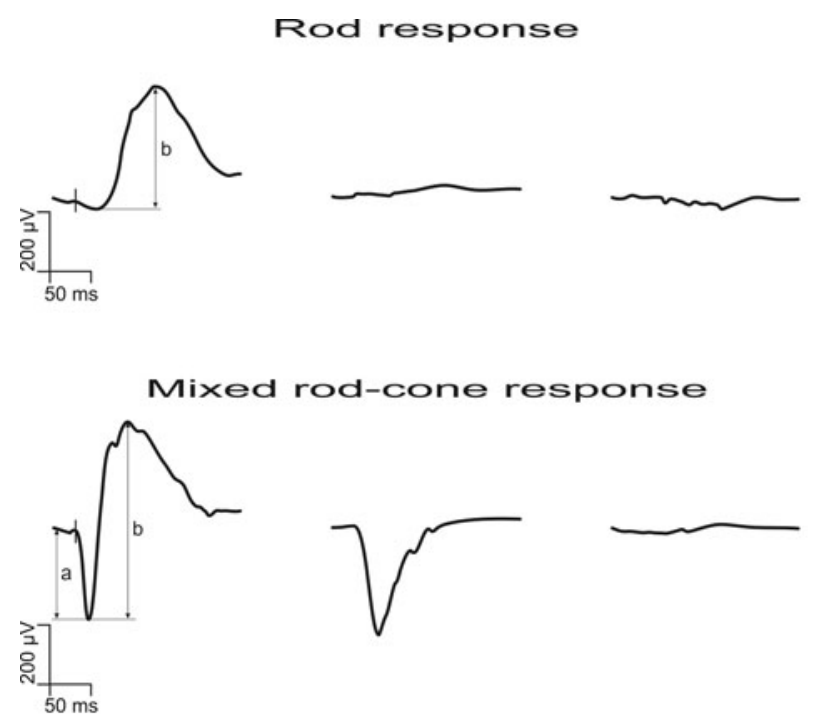

Cone response

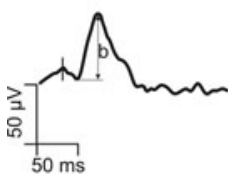

A

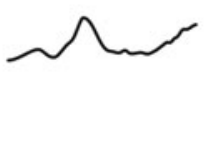

B

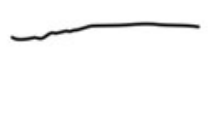

C
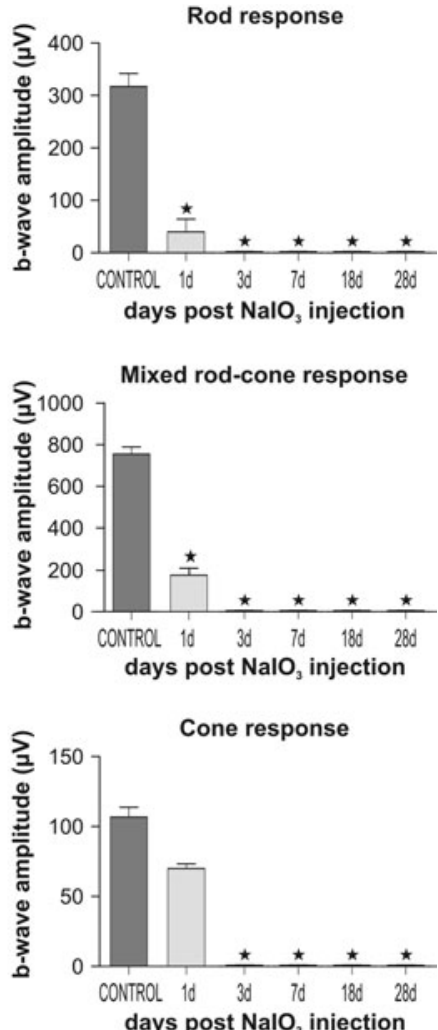

D

\section{Discussion}

In our study we provided new insight into morphological and functional retinal changes in response to $\mathrm{NaIO}_{3}$. Concurrently we supported previous reports documenting that the retinal pigment epithelium is the initial site of the toxic action of $\mathrm{NaIO}_{3}$, with secondary effects exerted on photoreceptors. The effect of $\mathrm{NaIO}_{3}$ on the RPE has been attributed to several mechanisms. Firstly, $\mathrm{NaIO}_{3}$ has been postulated to react with melanin, increasing its ability to convert glycine into glucoxylate, a potentially cell toxic compound [7]. Furthermore, $\mathrm{NaIO}_{3}$ has been demonstrated to inhibit various enzyme activities, for example triose phosphate dehydrogenase, lactate dehydrogenase, and 

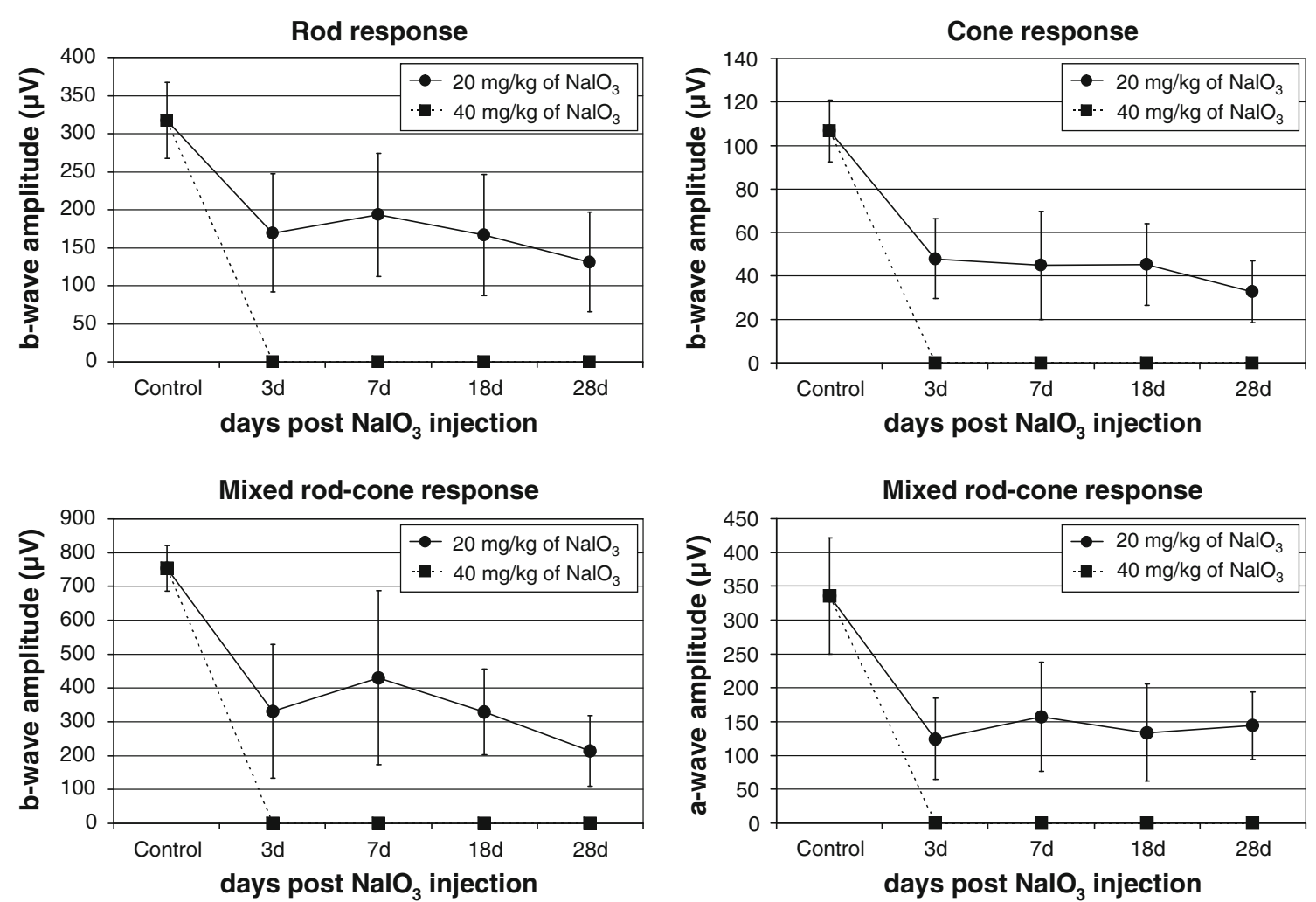

Fig. 5 A comparison of the b-wave and a-wave amplitudes after $\mathrm{NaIO}_{3}$ injection in the higher $(40 \mathrm{mg} / \mathrm{kg})$ and lower $(20 \mathrm{mg} / \mathrm{kg})$ doses over time. The higher dose suppressed the ERG response completely from the 3rd day post sodium iodate delivery while the lower

succinyl dehydrogenase, which play important roles in the process of energy production in cells [8]. Several reports have also documented alterations in the strength of adhesion between the sensory retina and the RPE due to $\mathrm{NaIO}_{3}$ intoxication [6,9]. Moreover, $\mathrm{NaIO}_{3}$ has been proven to break down the outer blood-retina barrier, exposing photoreceptor cells to its harmful influence from the choroidal circulation and accelerating the process of retinal degeneration [10].

Based on previous reports, RPE death following $\mathrm{NaIO}_{3}$ exposure has been attributed to the process of necrosis [11]. In contrast to apoptosis, necrosis is usually induced by massive physical or chemical injury. Moreover, it always affects groups of cells and is accompanied by inflammation. The results of our study confirmed the presence of severe injury in the RPE, indicating this model as a useful tool for studying various aspects of acute selective retinal damage. As early as the 1 st day after $\mathrm{NaIO}_{3}$ injection we observed widespread damage to RPE cells that was visible as massive necrosis along with kariolysis and melanin clumping. The extensive destruction of the RPE subsequently resulted in gradual changes in retinal morphology and the progressive degeneration of photoreceptors, causing their selective death in the process of apoptosis with a

concentration of $\mathrm{NaIO}_{3}$ led to a moderate decline in the a- and b-wave under both scotopic and photopic conditions. The results are presented as mean $\pm \mathrm{SD}$

peak intensity on the 3rd day after $\mathrm{NaIO}_{3}$ administration. This observation is consistent with studies on diseases resulting from defects in genes normally expressed in the RPE which lead to the degeneration of photoreceptors. Likewise, defects of genes expressed in photoreceptors have their consequence in RPE dysfunction. Such a causeand-effect relationship may clarify our observations and corroborate the thesis that the RPE and photoreceptors should be considered as a common functional unit due to their synergistic interaction.

In our study we observed that after exposure to the lower dose of $\mathrm{NaIO}_{3}$, necrotic as well as apoptotic changes within the RPE and photoreceptors were confined to the central retina. However, the damage induced by the higher dose of $\mathrm{NaIO}_{3}$ spread over both the central and peripheral parts of the retinal area. Moreover, we observed advanced apoptosis in the outer nuclear layer on the 3rd day after $\mathrm{NaIO}_{3}$ administration regardless of the dose used. This shows that different doses of $\mathrm{NaIO}_{3}$ can induce injury of similar severity to the photoreceptor layer, although with a different extent of damage. The observed regional pattern of photoreceptor degeneration induced by sodium iodate injection resembles the models found in other toxic compound-induced or inherited RPE disorders in which RPE 
cells are the primary site of pathology. Such models have been described for Royal College of Surgeons rats, vitiligo mice, and OAT-deficient mice $[12,13]$. In each of these pathological models, photoreceptors in the central retina were more severely affected than those located peripherally and there was relative sparing of the photoreceptors in the region directly adjacent to the ora serrata, similarly to our experimental data. Although the local variability of $\mathrm{NaIO}_{3}-$ induced retinal damage has been mentioned in previous reports [14], to our knowledge this is the first study providing a quantitative assessment of regional changes in the outer nuclear layer in response to two different $\mathrm{NaIO}_{3}$ doses. The severity of the damage observed in the central retina was similar regardless of the dose administered. However, no significant changes in either the RPE or photoreceptor morphology were observed in the peripheral retina after the injection of the lower dose of $\mathrm{NaIO}_{3}$ compared with the controls. The reason for this discrepancy is elusive and may result from some disparities in local vascular anatomy. Such a pathophysiological phenomenon could be partially explained by different metabolism of the cells residing in central and peripheral parts of the retina. On the other hand, it may be a consequence of an effective regenerative potential of the peripheral regions of the RPE. If such endogenic regeneration occurred, it would justify undertaking further investigations aimed at elucidating the pathomechanism involved.

In this experiment we also demonstrated that gradual RPE degeneration is accompanied by functional dysfunction of the neurosensory retina detected in electrophysiological tests. On the 1 st day after the higher dose of $\mathrm{NaIO}_{3}$ we observed a substantial decline in b-wave amplitude in scotopic ERG after white flash of low intensity, revealing extensive impairment of the rod pathway despite minor morphological changes in the neurosensory retina. Moreover, at this time point we noted a characteristic electronegative pattern in the scotopic bright-flash combined rod-cone response with a selective reduction of the b-wave to the extent that the peak of the b-wave failed to reach the baseline. Typically, a negative ERG waveform implies selective dysfunction of the middle retina with a relatively high preservation of photoreceptor function. However, negative ERGs have been reported in some patients with retinitis pigmentosa, although this disorder affects predominantly photoreceptors $[15,16]$. Furthermore, there have been reports showing that negative ERGs can be obtained from Royal College of Surgeons rats displaying a defect in phagocytosing function of the RPE, which is a counterpart of the human RP [17]. Several investigators have also demonstrated that a transient negative ERG in rats may be induced by $\mathrm{NaIO}_{3}$ injection [18].

This study showed for the first time the presence of a negative electroretinogram in $\mathrm{NaIO}_{3}$-treated mice. Our morphological observations along with previously published results [3] demonstrated that primary retinal changes in the $\mathrm{NaIO}_{3}$-induced retinal degeneration model are confined to the outer retina without considerable damage to the middle and inner retinal layers. A variety of hypotheses have been proposed to explain the mechanisms that may contribute to this paradox, including glutamate toxicity or ectopic synaptogenesis $[17,19,20]$. However, it is also possible that an electronegative ERG in the presence of severe rod system dysfunction may result from a relatively preserved dark-adapted cone system function and does not indicate inner retinal rod system impairment [21]. Hence we suggest that the electronegative waveform recorded by a bright flash in a dark-adapted state is a consequence of maintained cone function accompanying rod system degeneration. Our assumption may be confirmed by the relatively stable $b$-wave detected in the photopic singleflash cone response on the 1st day after the injection of the higher dose of $\mathrm{NaIO}_{3}$, elicited under the rod suppression conditions. The observation is consistent with a recently published report indicating that the retinal toxicity induced by a very high dose $(50 \mathrm{mg} / \mathrm{kg})$ of $\mathrm{NaIO}_{3}$ was expressed primarily in rods, followed by cones [22]. Nevertheless, the complete RPE disintegration and subsequent acute photoreceptor degeneration observed on the 3rd day after exposure to our high concentration of $\mathrm{NaIO}_{3}$ resulted in total suppression of both scotopic and photopic retinal function. This indicates advanced functional damage to the whole retinal area.

In contrast to the higher dose, the lower dose of $\mathrm{NaIO}_{3}$ led to detectable impairment of retinal function and to declines in b-wave and a-wave ERG amplitudes under both scotopic and photopic conditions, but not to their complete extinction. It seems reasonable to suppose that certain areas within the retina can remain less damaged and retain their function. Based on our findings, we assume that the peripheral retina is responsible for the maintained retinal response after the lower dose of $\mathrm{NaIO}_{3}$, which is shown in ERG recordings.

In conclusion, the time course of pathological changes in the retinal pigment epithelium and photoreceptors in response to $\mathrm{NaIO}_{3}$ seems to be consistent with the loss of retinal function revealed in electrophysiological tests. Our comprehensive analysis concerning an $\mathrm{NaIO}_{3}$ dose-depend tissue response clearly indicates that the peripheral region of the retina reveals better resistance to sodium iodateinduced injury than its central part. Overall, we performed for the first time the morphometric analysis as well as evaluation of intensity of programmed cell death within different retinal regions. According to our findings, $\mathrm{NaIO}_{3}$ damages mostly the central pole of the retina and, depending on the dose, can also affect its peripheral area. Such a pathological phenomenon resembles those observed 
in some acquired degenerative disorders (age-related macular degeneration, toxic retinopathies e.g. chloroquine, thioridazine, or chloropromazine intoxications) in their clinical course that initially involve the central part of the retina and spread gradually on its peripheral region. Therefore, our results largely indicate that chemical damage induced by $\mathrm{NaIO}_{3}$ mimics some retinal degenerative diseases in humans and may serve as a model useful for studying retinal damage.

Acknowledgments The work was supported by European Union structural funds-Innovative Economy Operational Program POIG.01.01.02-00-109/09-00.

Open Access This article is distributed under the terms of the Creative Commons Attribution Noncommercial License which permits any noncommercial use, distribution, and reproduction in any medium, provided the original author(s) and source are credited.

\section{References}

1. Strauss O (2005) The retinal pigment epithelium in visual function. Physiol Rev 85:845-881 Review

2. Kaplan J, Bonneau D, Frézal J, Munnich A, Dufier JL (1990) Clinical and genetic heterogeneity in retinitis pigmentosa. Hum Genet 85:635-642

3. Franco LM, Zulliger R, Wolf-Schnurrbusch UE, Katagiri Y, Kaplan HJ, Wolf S, Enzmann V (2009) Decreased visual function after patchy loss of retinal pigment epithelium induced by lowdose sodium iodate. Invest Ophthalmol Vis Sci 50:4004-4010

4. Korte GE, Gerszberg T, Pua F, Henkind P (1986) Choriocapillaris atrophy after experimental destruction of the retinal pigment epithelium in the rat. A study in thin sections and vascular casts. Acta Anat (Basel) 127:171-175

5. Nilsson SE, Knave B, Persson HE (1977) Changes in ultrastructure and function of the sheep pigment epithelium and retina induced by sodium iodate. III. Delayed effects. Acta Ophthalmol (Copenh) 55:1027-1043

6. Yoon YH, Marmor MF (1993) Retinal pigment epithelium adhesion to Bruch's membrane is weakened by hemicholinium-3 and sodium iodate. Ophthalmic Res 25:386-392

7. Baich A, Ziegler M (1992) The effect of sodium iodate and melanin on the formation of glyoxylate. Pigment Cell Res 5:394-395
8. Enzmann V, Row BW, Yamauchi Y, Kheirandish L, Gozal D, Kaplan HJ, McCall MA (2006) Behavioral and anatomical abnormalities in a sodium iodate-induced model of retinal pigment epithelium degeneration. Exp Eye Res 82:441-448

9. Ashburn FS Jr, Pilkerton AR, Rao NA, Marak GE (1980) The effects of iodate and iodoacetate on the retinal adhesion. Invest Ophthalmol Vis Sci 19:1427-1432

10. Korte GE, Reppucci V, Henkind P (1984) RPE destruction causes choriocapillary atrophy. Invest Ophthalmol Vis Sci 25:1135-1145

11. Kiuchi K, Yoshizawa K, Shikata N, Moriguchi K, Tsubura A (2002) Morphologic characteristics of retinal degeneration induced by sodium iodate in mice. Curr Eye Res 25:373-379

12. Nir I, Ransom N, Smith SB (1995) Ultrastructural features of retinal dystrophy in mutant vitiligo mice. Exp Eye Res 61:363-377

13. Wang T, Milam AH, Steel G, Valle D (1996) A mouse model of gyrate atrophy of the choroid and retina. Early retinal pigment epithelium damage and progressive retinal degeneration. J Clin Invest 97:2753-2762

14. Mizota A, Adachi-Usami E (1997) Functional recovery of retina after sodium iodate injection in mice. Vision Res 37:1859-1865

15. Cideciyan AV, Jacobson SG (1993) Negative electroretinograms in retinitis pigmentosa. Invest Ophthalmol Vis Sci 34:3253-3263

16. Renner AB, Kellner U, Cropp E, Foerster MH (2006) Dysfunction of transmission in the inner retina: incidence and clinical causes of negative electroretinogram. Graefes Arch Clin Exp Ophthalmol 244:1467-1473

17. Bush RA, Hawks KW, Sieving PA (1995) Preservation of inner retinal responses in the aged Royal College of Surgeons rat. Evidence against glutamate excitotoxicity in photoreceptor degeneration. Invest Ophthalmol Vis Sci 36:2054-2062

18. Tanaka M, Machida S, Ohtaka K, Tazawa Y, Nitta J (2005) Third-order neuronal responses contribute to shaping the negative electroretinogram in sodium iodate-treated rats. Curr Eye Res 30:443-453

19. Lucas DR, Newhouse JP (1957) The toxic effects of sodium L-glutamate on the inner layers of the retina. AMA Arch Ophthalmol 58:193-201

20. Peng YW, Senda T, Hao Y, Matsuno K, Wong F (2003) Ectopic synaptogenesis during retinal degeneration in the royal college of surgeons rat. Neuroscience 119:813-820

21. Audo I, Robson AG, Holder GE, Moore AT (2008) The negative ERG: clinical phenotypes and disease mechanisms of inner retinal dysfunction. Surv Ophthalmol 53:16-40

22. Yamashita H, Yamasaki K, Sugihara K, Miyata H, Tsutsumi S, Iwaki Y (2009) Full-field electroretinography obtained using a contact lens electrode with built-in high-intensity white-lightemitting diodes can be utilized in toxicological assessments in rats. Ophthalmic Res 42:15-20 